\title{
Sexualidad en el adulto mayor, conductas y retos - revisión de la literatura
}

\section{Sexuality in the Elderly, Behaviors and Challenges - Review of the Literature}

\author{
Hugo López-Ramos ${ }^{1}$ Mauricio Medina-Rico ${ }^{2}$ Paula Hernandez ${ }^{3} \quad$ José Miguel Silva ${ }^{1}$
} ${ }^{1}$ Departamento de Urología, Facultad de Medicina, Pontificia
Universidad Javeriana, Bogotá, Colombia
2 Departamento de Epidemiología Clínica y Bioestadística,
Facultad de Medicina, Pontificia Universidad Javeriana,
Bogotá, Colombia
${ }^{3}$ Estudiante 4to año de Medicina, Pontificia Universidad Javeriana,
Bogotá, Colombia

Urol Colomb 2019;28:121-129.

\begin{abstract}
Address for correspondence Mauricio Medina-Rico, MD, MSc, Departamento de Epidemiología Clínica y Bioestadística, Cr. 7 \#40-62, Hospital Universitario de San Ignacio $2^{\circ}$ piso, Bogotá, Colombia (e-mail: medina.mauricio@javeriana.edu.co).
\end{abstract}

\section{Resumen}

\section{Palabras Clave \\ - sexualidad \\ - conducta sexual \\ - adulto mayor \\ - sexología \\ - andrología \\ - revisión}

Introducción Pese a que se ha descrito la presencia de actividad sexual en el adulto mayor, ella tiende a menospreciarse. Al no contar con personas con quienes puedan hablar de sus dificultades o les facilite información, los adultos mayores pueden tener problemas para tener una vida sexual activa, así como mayor probabilidad de presentar conductas de riesgo.

Objetivo Identificar las actividades sexuales y los problemas en ese campo más frecuentes en el adulto mayor.

Métodos Se realizó una revisión en las bases de datos: Medline, Embase, Lilacs y Scielo. Se incluyeron estudios que brindaron información sobre la sexualidad en el adulto mayor al respecto de: Conductas sexuales más frecuentes, condiciones asociadas a una mejor o peor satisfacción sexual, y comunicación con el adulto mayor. Resultados Se revisaron 2.342 artículos por título y el resumen. En total se seleccionaron 27 referencias para revisión en texto completo. Se identificó la importancia de la sexualidad en el adulto mayor en la calidad de vida y cómo ella se ve afectada por la presencia de condiciones como: cáncer, diabetes y depresión; además de falta de deseo, disfunción eréctil en el hombre y la menopausia en la mujer. Dentro de las conductas sexuales más frecuentes se describen: los besos, el coito genital, el sexo oral y la masturbación.

Conclusiones Es importante conocer las condiciones que afectan la sexualidad del adulto mayor y entender la forma en que ellos desean vivir su sexualidad para facilitar la comunicación en la consulta y ayudarlos a tener una vida sexual satisfactoria. received

October 18, 2017

accepted

February 19, 2018
DOI https://doi.org/

10.1055/s-0038-1645849.

ISSN 0120-789X.

eISSN 2027-0119.
Copyright ( 2019, Sociedad Colombiana License terms de Urología. Publicado por Thieme Revinter Publicações Ltda., Rio de Janeiro, Brazil. Todos los derechos reservados.

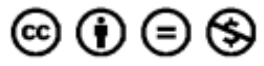




\begin{abstract}
Keywords

- sexuality

- sexual behavior

- aged

- sexology

- andrology

- review

Introduction Although the presence of sexual activity in the elderly has been described, it tends to be underestimated. By not having people with whom they can talk about their difficulties or providing them with information, older adults may have problems to have an active sex life, as well as a greater probability of having risky behaviors.

Aim To identify the most frequent sexual activities and problems in this field in the elderly.

Methods A review was performed in the databases: Medline, Embase, Lilacs and Scielo. We included studies that provided information about sexuality in the older adult regarding: More frequent sexual behaviors, conditions associated with better or worse sexual satisfaction, and communication with the elderly.

Results 2,342 articles were reviewed by title and abstract. In total, 27 references were selected for full-text review. The importance of sexuality in the elderly was identified in the quality of life and how it is affected by the presence of conditions such as: cancer, diabetes and depression; In addition to lack of desire, erectile dysfunction in men and menopause in women. Among the most frequent sexual behaviors are described: kissing, genital intercourse, oral sex and masturbation.

Conclusions It is important to know the conditions that affect the sexuality of the older adult and to understand the way in which they want to live their sexuality, to facilitate the communication in the consultation and to help them to have a satisfactory sexual life.
\end{abstract}

\section{Introducción}

A medida que el cuerpo humano envejece, el deterioro progresivo de los sistemas se hace evidente, hasta comprometer las actividades diarias entre ellas, la sexualidad. ${ }^{1}$ Sin embargo, la sexualidad continúa siendo una esfera natural y altamente deseada entre los adultos mayores, ${ }^{2,3}$ estando, entre otras cosas, relacionada con una mejor calidad de vida en esa población. ${ }^{4}$

A pesar de ser un tema importante en ese grupo etario, es con frecuencia visto como tabú, razón por la que tiende a ignorarse la sexualidad de las personas mayores; esa visión del tema en ocasiones es reforzada por barreras culturales que limitan una discusión abierta tanto con profesionales de la salud, como con la pareja y con los familiares. ${ }^{5,6}$ De esa manera, el adulto mayor que desea mantener una vida sexual activa puede percibir un acceso limitado a la información. ${ }^{5,7}$ Eso puede ocasionar diferentes dificultades, siendo que la persistencia del impulso sexual en el adulto mayor puede ser una fuente potencial de conductas de riesgo, de no orientarse correctamente. $^{8}$

Aunque en el adulto mayor algunas condiciones como la fragilidad y el deterioro cognitivo pueden verse asociadas en el compromiso de la vida sexual, ${ }^{9,10}$ el sexo genital, el sexo oral y la masturbación se han visto descritas dentro de las prácticas sexuales de esa población ${ }^{11,12}$; sin embargo, siendo los besos y las caricias las manifestaciones sexuales más frecuentemente mencionadas cuando las otras prácticas sexuales se ven comprometidas. ${ }^{11,13}$ Con una frecuencia para la actividad sexual variable, autores como Lee y col. (2016) han descrito una frecuencia de 20 más veces al mes. ${ }^{11}$ En nuestro medio en un estudio realizado por Silva y col. (2006) se evidenció que más del $50 \%$ de los adultos mayores de 70 años tienen relaciones sexuales regulares con menor presencia de deseo sexual en mujeres; sin embargo, con un grado de satisfacción sexual similar entre hombres y mujeres. ${ }^{14}$

Dada la creciente importancia del tema especialmente con relación en el aumento de la expectativa de vida, las condiciones de salud general en la población adulta mayor y el posible desconocimiento del personal de salud en esos temas, es necesario buscar y sintetizar la evidencia dirigida al entendimiento de la sexualidad en las personas mayores en cuanto a: 1 . las prácticas sexuales más frecuentes en el adulto mayor, 2. las condiciones físicas o psicosociales asociadas a un deterioro de la vida sexual, diferentes de la disfunción eréctil o la menopausia, siendo que esas ya están ampliamente descritas en hombres y mujeres respectivamente; y 3. cómo se está dando la información al adulto mayor o sus familiares en esos temas, que pueda ayudarles a tener una vida sexual satisfactoria.

\section{Métodos}

Se realizó una revisión de la literatura en 4 bases de datos (Medline, Embase, Lilacs y Scielo). Se establecieron los criterios de búsqueda en forma de texto libre y términos indexados. Para caracterizar sexualidad se utilizaron los 
términos libres: "Sexual Behavior," “sexuality," "sexual desire" y "sex counseling"; y los términos indexados: "Sexuality" y "Sexual Behavior." Para definir el concepto de adulto mayor se incluyeron los términos en texto libre: "older people" y "older adult"; y los términos indexados: "Aged, 80 and over" y "Aged." Como restricción se limitó la búsqueda a publicaciones en los últimos 10 años y filtro por edad para personas mayores de 65 años. También se hizo una búsqueda en literatura gris en las páginas del National Technical Information Service (NTIS) y el European Association for Gray Literature Exploitation (EAGLE), en las cuales no encontramos información relevante.

Los artículos debían ser estudios originales. Para describir las prácticas sexuales se consideraron los estudios que evaluaron al adulto mayor sano o con comorbilidades que no afectaran de manera importante la salud del individuo tales como el cáncer; o que tuvieran alguna comorbilidad directamente asociada a un deterioro de la vida sexual como la disfunción eréctil o la menopausia.

Para evaluar las comorbilidades asociadas a un compromiso de la sexualidad del adulto mayor se consideraron principalmente estudios retrospectivos que describieran la posible asociación entre las diferentes condiciones y el deterioro de la vida sexual, por lo cual estudios que estuvieran dirigidos al manejo de condiciones específicas como: Disfunción eréctil, eyaculación rápida, o enfermedades neurodegenerativas, fueron excluidos.

Para evaluar los artículos que mencionaran la comunicación sobre temas de sexualidad con el adulto mayor se tuvieron en cuenta estudios cualitativos $\mathrm{y}$ estudios transversales donde se indagaran los temas que se están abarcando en la consulta, qué temas consideran pacientes o médicos necesarios a tratar durante la misma, y qué posibles preferencias tienen las personas mayores de edad al momento de transferir dicha información.

Las referencias fueron revisadas por título y el resumen, por dos revisores de forma independiente. A partir de la primera selección de artículos, se revisaron las referencias en texto completo asegurando que ellos dieran algún dato de interés mencionado previamente sobre la sexualidad en el adulto mayor para la elaboración la revisión. Se eliminaron los artículos duplicados, y se ignoraron los estudios que estuvieran escritos en un idioma diferente al inglés o al español.

\section{Resultados}

La búsqueda arrojó un total de 2.342 referencias, que fueron revisadas por título y resumen. En total, se seleccionaron 74 referencias para revisión en texto completo, de las cuales se excluyeron 46 porque no eran artículos originales, no expresaban datos de interés, o eran referencias que no tenían disponible texto completo (formato de poster o resumen). Se analizaron finalmente 27 artículos en texto completo para la extracción de datos (-Fig. 1).

\section{Conductas Sexuales en el Adulto Mayor}

Se encontraron ocho estudios al respecto. Seis artículos mencionaban la sexualidad como un componente aún presente en los adultos mayores, siendo más frecuentemente observado en hombres que en mujeres. ${ }^{1,11-13,15,16}$ PalaciosCena y col. (2012), mencionan una proporción del 62,3\% de los hombres y un $37,4 \%$ de las mujeres que se mantienen sexualmente activas en la vejez, proporción que disminuye después de los 75 años ${ }^{1}$; no obstante, otros autores describen una proporción inclusive mayor de vida sexual activa en esa población, Wang y col. (2015) encontraron que un 79\% de hombres y un $76 \%$ de mujeres habían tenido actividades sexuales en los últimos 12 meses. ${ }^{16}$ Cuatro estudios mencionaban la frecuencia con la cual se ve la actividad sexual en el adulto mayor; todos los estudios describiendo hallazgos similares con una frecuencia de dos relaciones sexuales al mes. ${ }^{1,11-13}$

Seis estudios hicieron mención a las prácticas sexuales más frecuentes en los adultos mayores. Autores como Shkolnik y col. (2013), Lee y col. (2016) y Palacios-Cena y

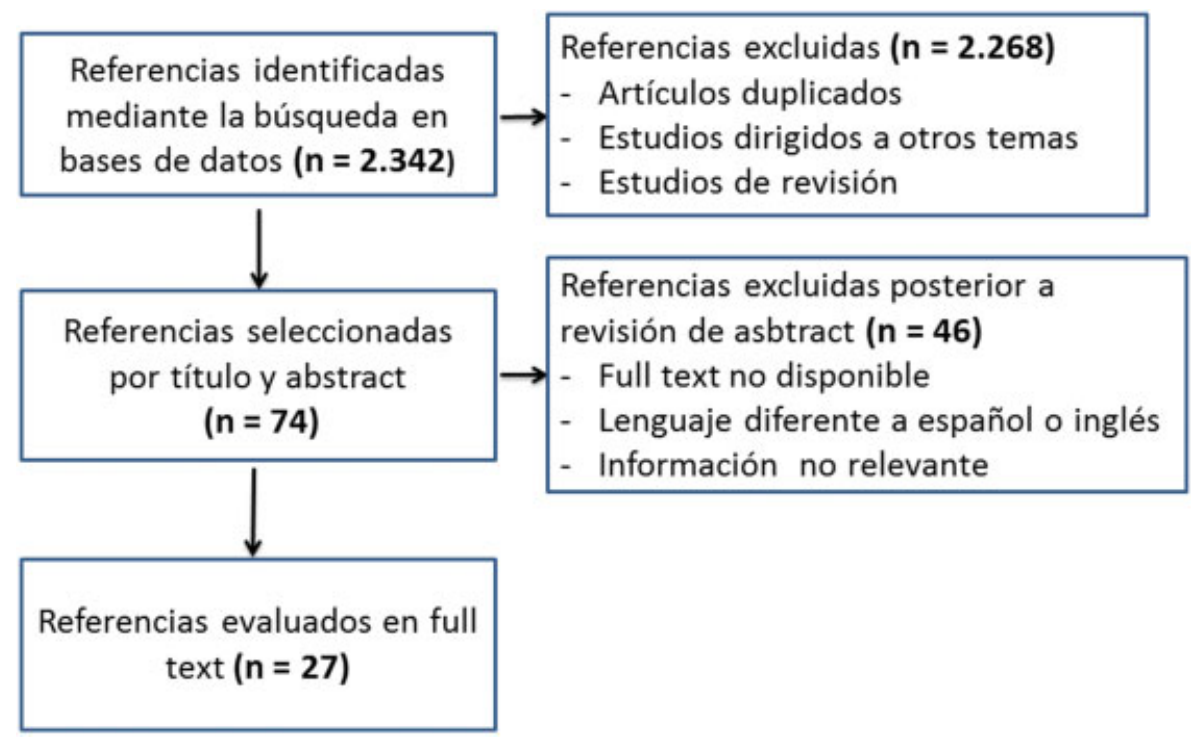

Fig. 1 Flujograma de la búsqueda. Selección y elegibilidad de estudios. Proceso y criterios de selección de artículos. 
col. (2012) identificaron a los besos y las caricias como las prácticas sexuales más frecuentes tanto en hombres como en mujeres, encontrándose en un $78,3 \%$ de ellos, seguido del coito genital y la masturbación llegando a presentarse en el $17 \%$ de hombres y el $7 \%$ de las mujeres y, finalmente, el sexo oral. ${ }^{1,11,13}$ Pese a todo eso, otros autores como Gledhill y col. (2014) y Fileborn y col. (2015) describen que aunque la masturbación y otras prácticas sexuales como besos y caricias pueden ser bastante frecuentes, el sexo coital sigue siendo preferido de ser posible. ${ }^{3,17}$

Por su parte, Trompeter y col. (2012) describe la satisfacción de la vida sexual de las mujeres mayores, encontrando que el $61 \%$ de ellas se sienten satisfechas con su sexualidad y el $78 \%$ de las mujeres con vida sexual activa, se sienten satisfechas con la cercanía emocional que tienen con sus parejas durante el sexo. ${ }^{15}$ ( - Tabla $\mathbf{1}$ ).

\section{Comorbilidades de Disfunción Sexual}

Se encontraron 19 estudios al respecto. Mazo y col. (2011), describen la relación entre la satisfacción sexual en el adulto mayor y una buena calidad de vida ${ }^{4}$; así mismo, Galinsky y col. (2014) lo asocian con una mejor percepción en la calidad de los matrimonios. ${ }^{18}$ Hartmans y col. (2015) comentan una relación de la cognición general y la memoria de las personas, así como la inteligencia con una mejor sexualidad. ${ }^{10}$ Por otro lado, Thompson y col. (2011), describen una asociación con el estado físico del individuo o de la pareja teniendo en cuenta que una mayor satisfacción sexual implica un buen estado de salud que incluye tanto el aspecto físico como el mental. ${ }^{19} \mathrm{En}$ eso último, autores como Lonnèe-Hoffmann y col. (2014) y Hyde y col. (2012) describen las condiciones en salud mental como factores asociados a alteraciones de la sexualidad, principalmente siendo los trastornos depresivos los más influyentes. ${ }^{20,21}$

Se han descrito diferentes comorbilidades asociadas al compromiso de la vida sexual del adulto mayor; Wang y col. (2015) mencionan que más del 30\% de los adultos mayores llegan a referir dificultades durante la actividad sexual, asociadas a la disminución del deseo. ${ }^{16}$ Así mismo, Gledhil y col. (2014) sugieren que la presencia de antecedentes en salud física como diabetes puede ser un limitante para una vida sexual satisfactoria ${ }^{17}$; de eso último autores como Lynea y col. (2013) describen una relación negativa de la sexualidad del adulto mayor con antecedentes como: hipercolesterolemia, diabetes, hipertensión arterial, disminución en la visión, cuidado crónico de heridas, patologías de vejiga, problemas gastrointestinales, cirugías mayores y problemas osteoarticulares. ${ }^{22}$ Otros autores como Bach y col. (2013) refieren una disminución en la actividad sexual en los adultos mayores con dolor, compromiso del estado energético o de movilidad. ${ }^{22}$ Así mismo, Lee y col. (2013), encontraron una relación entre fragilidad y un menor funcionamiento sexual entendiendo fragilidad como: cansancio, sarcopenia y baja actividad física. ${ }^{9}$

Así mismo, se han descrito situaciones que afectan la sexualidad del adulto mayor como: ser fumador, tener alguna demencia y falta de apoyo emocional. ${ }^{22}$ Por otra parte, dentro de las condiciones expresamente relacionadas con la sexualidad Hughes y col. (2015) identificaron la disminución de la libido, la lubricación inadecuada como factores asociados a una peor actividad sexual en el adulto mayor. ${ }^{23}$ Igualmente, Adeoti y col. (2015), mencionan dificultades como la dispareunia en las mujeres y dificultades en la eyaculación en los hombres hasta en un $57,7 \%{ }^{6}$

Se encontraron cuatro estudios que describieron factores positivos para una mejor vida sexual en el adulto mayor. Valadares A y col. (2014), describen una asociación entre salud mental y física con una mejor vida sexual, igualmente encontrando esa asociación con una mayor educación y mejores ingresos económicos. ${ }^{24}$ No obstante, otros autores como Momtaz YA y col. (2014), pese a referir resultados similares en cuanto el nivel educativo de los individuos, no encontraron asociación con los ingresos económicos la actividad sexual. ${ }^{25}$ Por otro lado, los autores Galinsky W y col. (2014) y Flynn G y col. (2015), mencionan la importancia de una pareja igualmente saludable, encontrando menor actividad sexual en personas sin una pareja estable. ${ }^{18,26}$

Cuatro estudios mencionan algunos factores positivos que se encuentran asociados para una vida sexual satisfactoria en el adulto mayor entre los que se destacan: tener una función cognitiva preservada, tener una buena percepción de imagen corporal, tener una buena salud mental y física, y tener una pareja estable 1 13,15,20; de ese último Palacios-Cena y col. (2012), describen que no tener pareja es predictor de poca actividad sexual OR 5,79 (95\% CI 3.98-8.42) en hombres y 12 (IC95\%, 8,4-17,2) en mujeres. ${ }^{1}$

Finalmente el estudio de Iveniuk J y col. (2016), evaluaron la influencia de la religión dentro de la sexualidad encontrando que ella no afecta la vida sexual del adulto mayor; por el contrario, aquellas personas con algún componente religioso en sus vidas se sienten más complacidos con la vida sexual que tienen con sus parejas. ${ }^{2}$

\section{Comunicación con el Adulto Mayor}

Se encontraron seis artículos al respecto. Tres estudios mencionaban la perspectiva de los pacientes al momento de hablar de sexo con los médicos u otras personas. Los estudios de Ama y col. (2013) y Farrel y col. (2012) describen la incomodidad que siente el adulto mayor al momento de hablar de sexo. Ama y col. describen que cerca de la tercera parte de la población, se puede sentir incómoda de hablar de sexo con sus parejas. Así mismo, Farrel y col. encuentran un proporción de incomodidad menor cuando se discute de sexo con un médico llegando a ser del 2,2\%, y aumentando si está presente un familiar en la consulta. ${ }^{5,7}$ Así mismo, Hirayama y col. (2011), describen que la incomodidad que se presenta a la hora de hablar de sexo no es diferente entre personal médico y conocidos, no obstante rescatando que hablar de los problemas sexuales del paciente puede ayudar a disminuir el estrés producto de los mismos. ${ }^{27}$

Dos estudios mencionaban la comunicación de conductas de riesgo en el adulto mayor. Zhou y col. (2014) describen en la muestra de su estudio como el $100 \%$ de los individuos refirieron alguna conducta de riesgo recientemente: pagar por sexo, relaciones no protegidas, relaciones con parejas no 


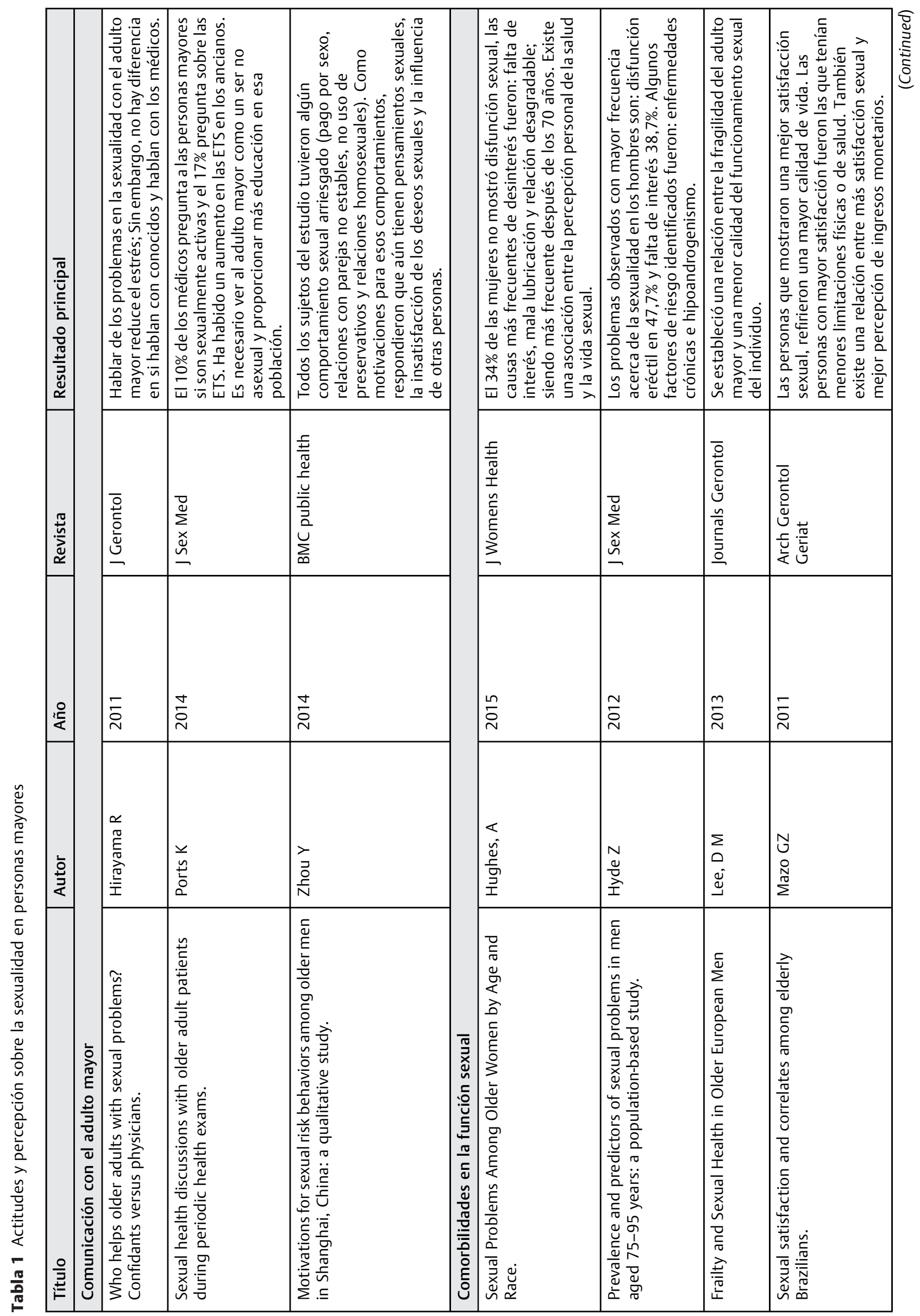




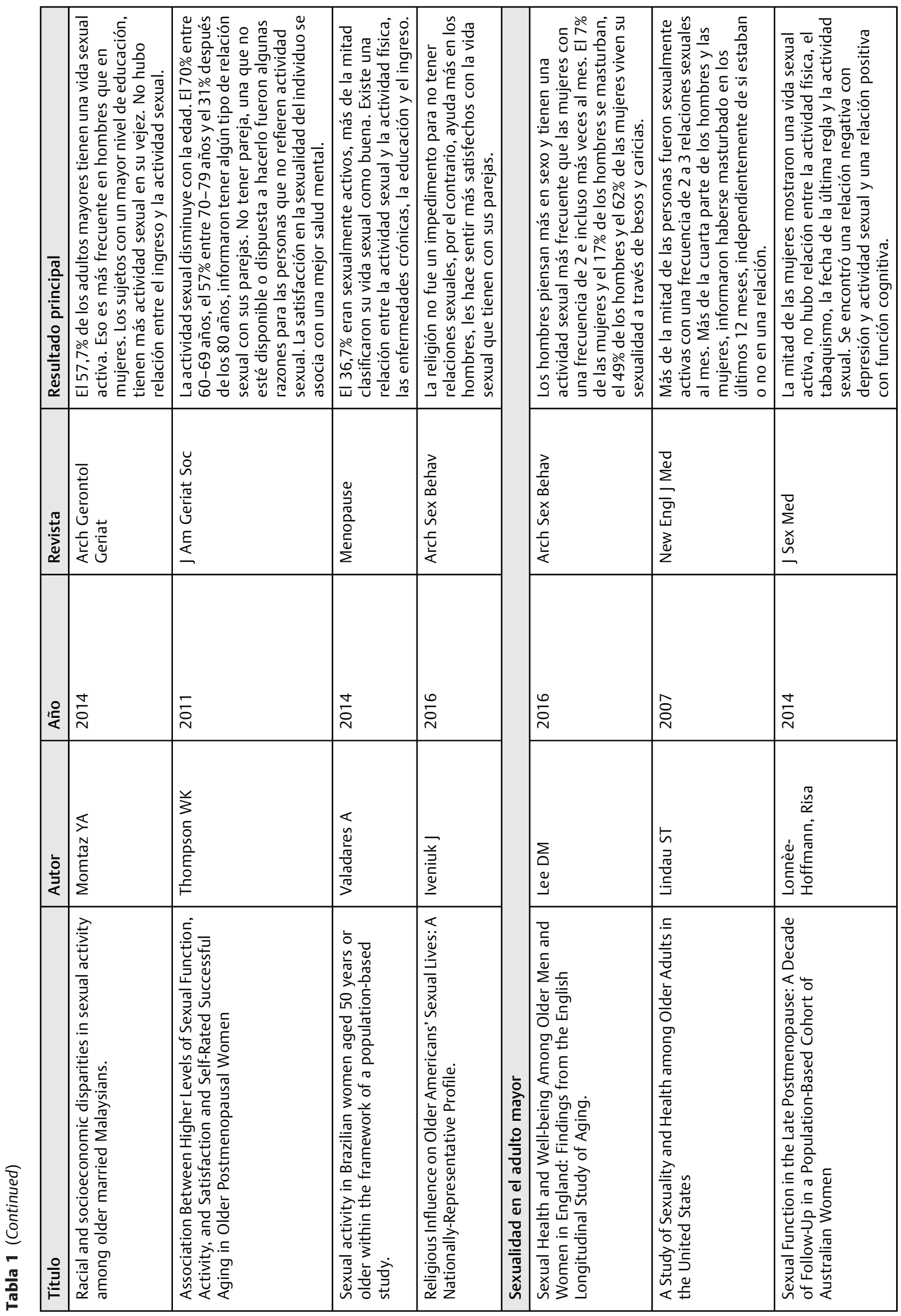




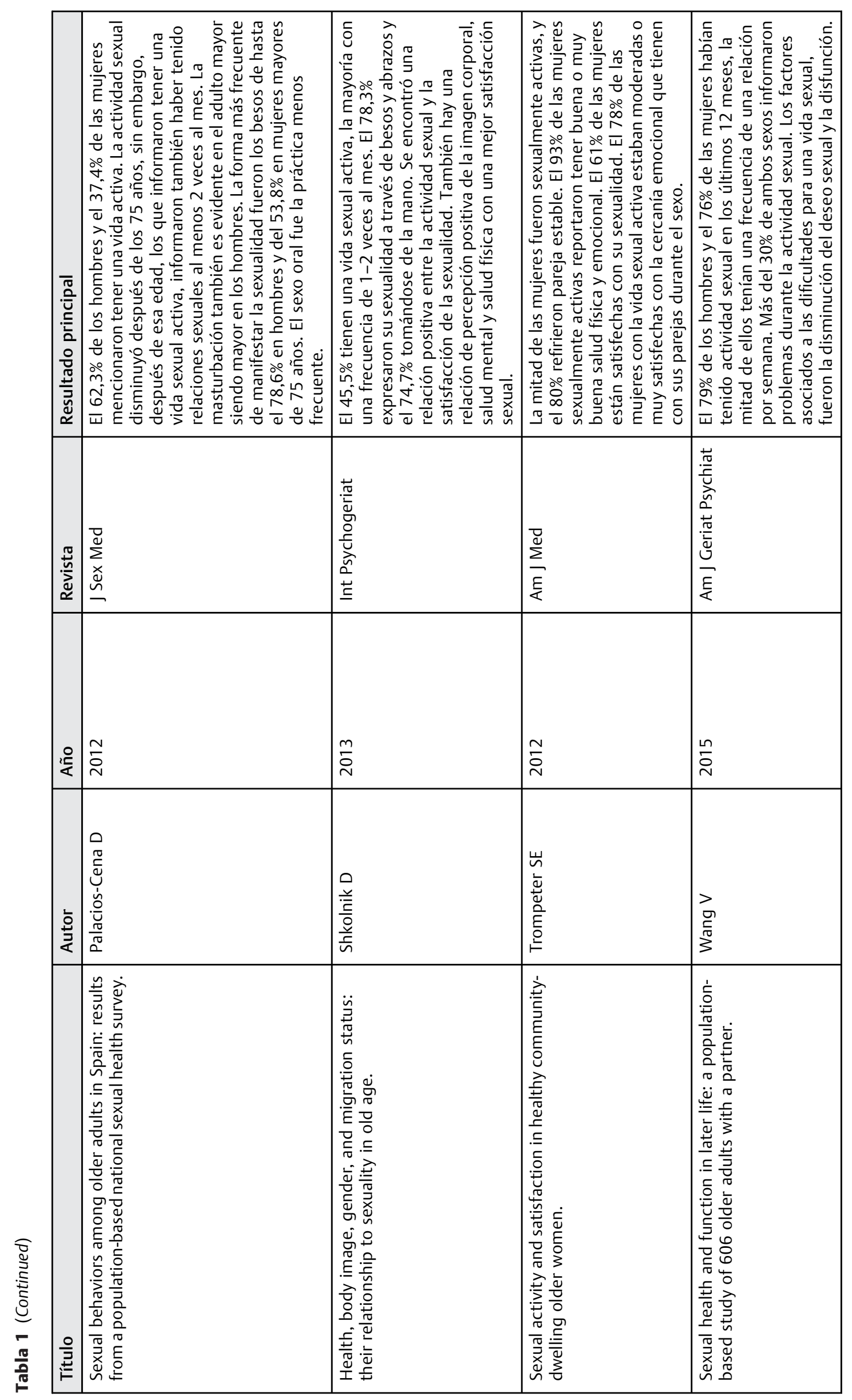


estables y relaciones con personas del mismo sexo. ${ }^{8}$ Por otro lado, Ports y col. (2014), encontraron que solo el $10 \%$ de los médicos preguntan a los adultos mayores en las consultas si son personas sexualmente activas, así mismo solo el $17 \%$ preguntan sobre enfermedades de transmisión sexual. ${ }^{28}$

Finalmente, en el estudio de Clark y col. (2014), describen la comunicación con el adulto mayor desde la perspectiva del médico, haciendo énfasis en la importancia de dar información de esos temas a todas las personas durante la consulta y no esperar a que sean ellos los que inicien la conversación sobre sus problemas y necesidades bajo el supuesto de que pueden sentirse incómodos al ser abordados por el médico. ${ }^{29}$

\section{Discusión}

Dentro de los hallazgos de la revisión se observa que aproximadamente la mitad de hombres y mujeres adultos mayores son sexualmente activos. ${ }^{13}$ Igualmente, se pudo observar una asociación entre vida sexual activa y una mejor calidad de vida tanto a nivel personal como satisfacción con la pareja. ${ }^{13,18,26}$ Así mismo, se identificaron algunas condiciones asociadas a la disminución de la actividad sexual en el adulto mayor, diferentes de la disfunción eréctil en el hombre y la menopausia en las mueres como: la diabetes o la hipertensión, la fragilidad, la depresión y la falta de deseo. $^{12,22,30}$ Por otro lado, un adecuado nivel cognitivo, un mejor nivel educativo en la vida y una pareja estable parecieron ser factores facilitadores para tener una vida sexual activa sana. ${ }^{1,12}$

A pesar de que la actividad sexual disminuye con la edad, ella sigue presentándose en el adulto mayor. No obstante, ellos pueden tener dificultades a la hora de encontrar alguien con quien hablar llegando a sentirse incómodos con familiares e inclusive personal de salud dada la complejidad que puede representar ese tema. ${ }^{7,28}$ De la misma manera, familiares y médicos pueden no abarcar adecuadamente el tema con los pacientes mayores ante la incomodidad que puede representar el tema o por la creencia de que ellos son seres asexuales. ${ }^{28,29}$ Lo anterior ha producido un vacío en el conocimiento aumentando la probabilidad de que ellos caigan en conductas sexuales de riesgo. ${ }^{8}$

Esta revisión permite hacer un mapeo de la importancia de la sexualidad en el adulto mayor dejando que el lector reflexione sobre las dificultades que puede tener el adulto mayor para tener una vida sexual activa; al tiempo que ubica algunos de los desafíos que tiene el personal de salud para mejorar la comunicación con el individuo y ayudarlo a tener una vida sexual satisfactoria. ${ }^{5,29}$ Bajo dichas dificultades, se han descrito algunas pautas para facilitar la comunicación con el paciente acerca de su sexualidad tal como el modelo PLISSIT en donde se inicia pidiendo permiso para hablar del tema, se ofrece la respectiva información, se sugieren intervenciones para mejorar la vida sexual de la persona dependiendo de la causa o remitir a alguien que sepa del tema y finalmente ofrecer la terapia correspondiente. ${ }^{31}$ No obstante, pese a ser un tema que debe abarcarse con el paciente, es prioritario también educar a familiares sobre la existencia de dicha sexualidad en el adulto mayor para facilitar el abordaje del tema con el paciente. Además, se requiere la capacitación del personal de salud sobre los conocimientos y herramientas necesarios para responder las inquietudes del adulto mayor, situación que se podría trabajar desde la formación médica. ${ }^{6,25,31}$

Como fortalezas esta revisión cuenta con una búsqueda amplia de la literatura en diferentes bases de datos, una revisión pareada para corroborar la extracción de la información más reciente y relevante sobre el tema, y una búsqueda reproducible que permite al lector profundizar en el tema si así lo quiere. Dentro de las limitaciones del estudio radica que ella es una revisión narrativa y no una revisión sistemática, por lo cual no contamos con toda la información existente. Igualmente, los resultados obtenidos corresponden a una descripción de los hallazgos emanados de los diferentes estudios y no a una conclusión propia.

Finalmente, hallazgos similares han podido encontrare en otras revisiones donde se describen una proporción y frecuencia similares con que los adultos mayores tienen relaciones sexuales, las condiciones más frecuentemente asociadas y las consideraciones que debe tener el médico a la hora de hablar con el paciente ${ }^{32,33}$; en ese último campo, es necesario hacer evidente la necesidad de instruir a médicos y otras personas del área de la salud, como por ejemplo, trabajadores en hogares geriátricos, sobre importancia de la vida sexual del adulto mayor aumentando su confianza para hablar del tema, y así poder brindar a los pacientes la ayuda correspondiente. ${ }^{34}$

Con este estudio, contribuimos a la formación del personal de la salud creando conciencia sobre la existencia de la sexualidad en el adulto mayor, y brindando conocimiento sobre las dificultades más frecuentemente observadas, así como algunas pautas para mejorar la comunicación con ellos. Adicionalmente, este artículo otorga información que permitirá el desarrollo de futuros estudios para mejorar la atención con los pacientes mayores, así como de intervenciones de condiciones diferentes a la disfunción eréctil o a la menopausia, asociadas a las dificultades en la sexualidad de esa población.

\section{Responsabilidades Éticas}

Protección de personas y animales.

Los autores declaran que para esta investigación no se han realizado experimentos en seres humanos ni en animales.

Confidencialidad de los datos.

Los autores declaran que en este artículo no aparecen datos de pacientes.

Derecho a la privacidad y consentimiento informado. Los autores declaran que en este artículo no aparecen datos de pacientes.

Financiamiento

El artículo fue financiado por los autores del artículo. 


\section{Conflictos de Interés}

Los autores no refieren ningún conflicto de interés.

\section{Bibliografía}

1 Palacios-Ceña D, Carrasco-Garrido P, Hernández-Barrera V, Alonso-Blanco C, Jiménez-García R, Fernández-de-las-Peñas C. Sexual behaviors among older adults in Spain: results from a population-based national sexual health survey.J Sex Med 2012;9 (01):121-129

2 Iveniuk J, O'Muircheartaigh C, Cagney KA. Religious Influence on Older Americans' Sexual Lives: A Nationally-Representative Profile. Arch Sex Behav 2016;45(01):121-131

3 Fileborn B, Thorpe R, Hawkes G, Minichiello V, Pitts M. Sex and the (older) single girl: experiences of sex and dating in later life. J Aging Stud 2015;33:67-75

4 Mazo GZ, Cardoso FL. Sexual satisfaction and correlates among elderly Brazilians. Arch Gerontol Geriatr 2011;52(02):223-227

5 Ama NO, Ngome E. Challenges faced by older women in Botswana in accessing services that address sexual and reproductive health, and family planning needs, in Botswana. S Afr Fam Pract 2013;55 (03):281-288

6 Olatayo AA, Kubwa OO, Adekunle AE. Sexuality in Nigerian older adults. Pan Afr Med J 2015;22:315

7 Farrell J, Belza B. Are older patients comfortable discussing sexual health with nurses? Nurs Res 2012;61(01):51-57

8 Zhou Y, Ding Y, Gu K, Lu X, Gao M, He N. Motivations for sexual risk behaviors among older men in Shanghai, China: a qualitative study. BMC Public Health 2014;14:802

9 Lee DM, Tajar A, Ravindrarajah R, et al. Frailty and Sexual Health in Older European Men. Journals Gerontol Ser A Biol Sci. Med Sci 2013;68:837-844

10 Hartmans C, Comijs H, Jonker C. The perception of sexuality in older adults and its relationship with cognitive functioning. Am J Geriatr Psychiatry 2015;23(03):243-252

11 Lee DM, Nazroo J, O'Connor DB, Blake M, Pendleton N. Sexual Health and Well-being Among Older Men and Women in England: Findings from the English Longitudinal Study of Ageing. Arch Sex Behav 2016;45(01):133-144

12 Lindau ST, Schumm LP, Laumann EO, Levinson W, O'Muircheartaigh CA, Waite LJ. A study of sexuality and health among older adults in the United States. N Engl J Med 2007;357(08):762-774

13 Shkolnik D, lecovich E. Health, body image, gender, and migration status: their relationship to sexuality in old age. Int Psychogeriatr 2013;25(10):1717-1727

14 Silva JM, Ramírez DC. Comportamiento sexual en la población mayor de 70 años. Rev Urol Colomb. 2006;14:71-76

15 Trompeter SE, Bettencourt R, Barrett-Connor E. Sexual activity and satisfaction in healthy community-dwelling older women. Am J Med 2012;125(01):37-43.e1

16 Wang V, Depp CA, Ceglowski J, Thompson WK, Rock D, Jeste DV. Sexual health and function in later life: a population-based study of 606 older adults with a partner. Am J Geriatr Psychiatry 2015; 23(03):227-233
17 Gledhill S, Schweitzer RD. Sexual desire, erectile dysfunction and the biomedicalization of sex in older heterosexual men. J Adv Nurs 2014;70(04):894-903

18 Galinsky AM, Waite LJ. Sexual activity and psychological health as mediators of the relationship between physical health and marital quality. J Gerontol B Psychol Sci Soc Sci 2014;69(03):482-492

19 Thompson WK, Charo L, Vahia IV, Depp C, Allison M, Jeste DV. Association between higher levels of sexual function, activity, and satisfaction and self-rated successful aging in older postmenopausal women.J Am Geriatr Soc 2011;59(08):1503-1508

20 Lonnèe-Hoffmann RAM, Dennerstein L, Lehert P, Szoeke C. Sexual function in the late postmenopause: a decade of follow-up in a population-based cohort of Australian women. J Sex Med 2014;11 (08):2029-2038

21 Hyde Z, Flicker L, Hankey GJ, et al. Prevalence and predictors of sexual problems in men aged 75-95 years: a population-based study. J Sex Med 2012;9(02):442-453

22 Bach LE, Mortimer JA, VandeWeerd C, Corvin J. The association of physical and mental health with sexual activity in older adults in a retirement community. J Sex Med 2013;10(11):2671-2678

23 Hughes AK, Rostant OS, Pelon S. Sexual Problems Among Older Women by Age and Race. J Womens Health (Larchmt) 2015;24 (08):663-669

24 Valadares AL, Santos Machado VS, da Costa-Paiva LS, de Souza MH, Osis MJ, Pinto-Neto AM. Sexual activity in Brazilian women aged 50 years or older within the framework of a population-based study. Menopause 2014;21(03):295-300

25 Momtaz YA, Hamid TA, Ibrahim R, Akahbar SAN. Racial and socioeconomic disparities in sexual activity among older married Malaysians. Arch Gerontol Geriatr 2014;58(01):51-55

26 Flynn T-J, Gow AJ. Examining associations between sexual behaviours and quality of life in older adults. Age Ageing 2015; 44(05):823-828

27 Hirayama R, Walker AJ. Who helps older adults with sexual problems? Confidants versus physicians. J Gerontol B Psychol Sci Soc Sci 2011;66(01):109-118

28 Ports KA, Barnack-Tavlaris JL, Syme ML, Perera RA, Lafata JE. Sexual health discussions with older adult patients during periodic health exams. J Sex Med 2014;11(04):901-908

29 Clark RD, Williams AA. Patient preferences in discussing sexual dysfunctions in primary care. Fam Med 2014;46(02):124-128

30 Ceballos MDP, Álvarez Villarraga JD, Silva Herrera JM, Mantilla D, Uribe JF. Guía de disfunción eréctil - Sociedad Colombiana de Urología. Elsevier Doyma. 2015. p. 1-32.

31 Omole F, Fresh EM, Sow C, Lin J, Taiwo B, Nichols M. How to discuss sex with elderly patients. J Fam Pract 2014;63(04):E1-E4

32 Taylor A, Gosney MA. Sexuality in older age: essential considerations for healthcare professionals. Age Ageing 2011;40 (05):538-543

33 Ni Lochlainn M, Kenny RA. Sexual activity and aging. J Am Med Dir Assoc 2013;14(08):565-572

34 Mahieu L, Gastmans C. Older residents' perspectives on aged sexuality in institutionalized elderly care: a systematic literature review. Int J Nurs Stud 2015;52(12):1891-1905 